\title{
Quantitative Determination of Vitamin C Concentration of Common Edible Food Sources by Redox Titration Using Iodine Solution
}

\author{
Laxminandan Satpathy ${ }^{1, *} \mathbb{( D}$, Naganandini Pradhan 1 (D), Deeptimayi Dash ${ }^{1}$ (D), Priyanka Priyadarshini \\ Baral $^{2}$ (D), Siba Prasad Parida 1,* (D)
}

1 Department of Zoology, School of Applied Sciences, Centurion University of Technology and Management, Bhubaneswar, Odisha, 752050 India; laxminandansatpathy@gmail.com (L.S.); naganandinipradhan1997@gmail.com (N.P.); dashdeeptimayi98@gmail.com (D.D.); paridasp@gmail.com (S.P.P.);

2 Department of Zoology, Dhenkanal Autonomous College, Dhenkanal, Odisha, 759001 India; priyankapb198@ gmail.com (P.P.B.);

* Correspondence: laxminandansatpathy@gmail.com (L.S.); paridasp@gmail.com (S.P.P.);

Scopus Author ID 57197747626

Received: 26.09.2020; Revised: 2.01.2021; Accepted: 5.01.2021; Published: 17.01.2021

\begin{abstract}
Vitamin C is isolated in 1928 and associated with hemorrhagic disease scurvy. Vitamin C is crucial for the development and maintenance of connective tissues in humans. It plays an important role in maintaining healthy gums, helps in wound healing, bone formation, relief from a common cold, and several metabolic functions. It is an antioxidant that safeguards the cells from free radical damage. It is used as a curative agent in many diseases and disorders. This research article attempts to find different concentrations of vitamin $\mathrm{C}$ in common edible foodstuffs. The iodine redox titration method is used for vitamin $\mathrm{C}$ quantity evaluation. This study is based on the vitamin $\mathrm{C}$ quantity of 27 common edible food sources, including lemon (Citrus limon), lime (Citrus aurantiifolia), navel orange (Citrus sinensis), grapevine (Vitis vinifera), fox grape (Vitis labrusca), pomegranate (Punica granatum), apple (Malus domestica), kiwifruit (Actinidia deliciosa), Indian tamarind (Tamarindus indica), holy basil (Ocimum tenuiflorum), coriander (Coriandrum sativum), tomato (Solanum lycopersicum), carrot (Daucus carota), radish (Raphanus sativus), bitter gourd (Momordica charantia), garlic (Allium sativum), onion (Allium cepa), potato (Solanum tuberosum), aubergine (Solanum melongena), bell pepper (Capsicum annuum), pea (Pisum sativum), common bean (Phaseolus vulgaris), pumpkin (Cucurbita moschata), pawpaw (Carica papaya), cucumber (Cucumis sativus), cabbage (Brassica oleracea var. capitata), and cauliflower (Brassica oleracea var. botrytis), are evaluated. The highest quantity of vitamin C present in Indian tamarind, i.e., $89.44 \mathrm{mg} / 100 \mathrm{~g}$. This study is intended to use new vitamin C sources for nourishment.
\end{abstract}

Keywords: vitamin C; COVID-19; diseases; immunity; iodine solution; redox titration.

(C) 2020 by the authors. This article is an open-access article distributed under the terms and conditions of the Creative Commons Attribution (CC BY) license (https://creativecommons.org/licenses/by/4.0/).

\section{Introduction}

Vitamins are essential organic nutrients that are required for various biochemical and physiological processes in the human body. Vitamins are classified as water-soluble (B complexes and C) and fat-soluble (A, D, E, K) vitamins based on their solubility. Vitamin C was first isolated in the year 1923 by Szent-Gyorgyi and synthesized by Haworth and Hirst [1]. Vitamin $\mathrm{C}$ is also known as L-ascorbic acid [2]. It is required for the biosynthesis of collagen fibers, L-carnitine, and some neurotransmitters; vitamin $\mathrm{C}$ is involved in protein metabolism 
$[2,3]$. Collagen is an important component of connective tissue, which plays a vital role in wound healing. Vitamin $\mathrm{C}$ is also an essential physiological antioxidant [4], and it regenerates other antioxidants within the body, including vitamin $\mathrm{E}$ (alpha-tocopherol) [5]. Ongoing research is evaluating whether vitamin $\mathrm{C}$ restricting the damaging effects of free radicals through its antioxidant property. It might prevent or delay the development of certain cancers, cardiovascular disease, and other diseases in which oxidative stress plays a crucial role [6, 7]. In addition to its antioxidant activity and biosynthetic functions, vitamin $\mathrm{C}$ plays a vital role in immune function [5] by improving the absorption of nonheme iron [8], the form of iron that presents in plant-based foodstuffs. The deficiency of vitamin $\mathrm{C}$ causes scurvy, characterized by capillary fragility, connective tissue weakness, and lassitude [2, 3, 5, 9-12]. Pauling suggested that ingestion of 1-3 g of vitamin $\mathrm{C}$ effectively prevents the common cold [13]. Regular use of vitamin $\mathrm{C}$ supplements shortens the time span of the common cold but does not reduce the risks of contracting a cold except for a person undergoing heavy physical activity [14]. A novel human coronavirus has been recently identified, the severe acute respiratory syndrome (SARS) coronavirus (CoV) 2, which causes the fatal coronavirus disease 2019 (COVID-19) [15]. During the outbreak of SARS-CoV-1 in 2003, the use of vitamin C was suggested as a nonspecific treatment for severe viral respiratory tract infections [16-19]. In chick embryo tracheal organ cultures, vitamin $\mathrm{C}$ shows increased resistance to infection caused by an avian coronavirus [20]. Many patients with severe COVID-19 have raised levels of mediators interleukin- 6 and endothelin-1, which can be reduced by administering high doses of vitamin $C$ [15]. Numerous abstracts are showing that vitamin $C$ may affect the immune system by transforming the T lymphocyte and production of interferon [21, 22]. Some studies demonstrated that in guinea pigs maximum tensile strength of scar tissue was achieved after supplementing vitamin $\mathrm{C}$ due to biosynthesis and collagen accumulation [23]. In recent studies, it has been found that the supplementation of vitamin $C$ with vitamin $E$ relieves oxidative stress in the blood and tissues of diabetic aged rats by modulating the antioxidant status and lipid profile $[24,25]$. Many studies showed the beneficial effect of vitamin C against heavy metal toxicity. Lead is one of the common environmental pollutants in which vitamin C's protective role is extensively studied [26]. Oral supplementation of vitamin $\mathrm{C}$ with antipsychotic drugs reverses ascorbic acid levels, reduces oxidative stress, and enhances the brief psychiatric rating scale (BPRS) score. Hence, in amalgamation, both drugs can be used in the treatment of schizophrenia [27]. Curcumin might increase vitamin C's effect in protecting the function of endothelial cells through its antioxidant with hypoglycemic and hypolipidemic actions [28, 29]. Approximately $70 \%-90 \%$ of vitamin C is absorbed by the cells at moderate intakes of 30-180 $\mathrm{mg}$ /day. But, at doses above $1 \mathrm{~g} /$ day, vitamin $\mathrm{C}$ absorption is less than $50 \%$, and unmetabolized ascorbic acid is excreted in the urine [5]. However, results from pharmacokinetic studies suggest that orally administered doses of $1.25 \mathrm{~g} /$ day ascorbic acid produce mean peak plasma concentrations of vitamin $\mathrm{C}$ of $135 \mathrm{micromol} / \mathrm{L}$, which are about two times higher than those produced by consuming 200-300 mg/day ascorbic acid from vitamin C-rich foodstuffs [30]. Pharmacokinetic modeling indicates that even at higher doses, i.e., $3 \mathrm{~g}$ ascorbic acid taken every 4 hours would produce peak plasma vitamin C concentrations of 220micromol/L [30]. The total vitamin $\mathrm{C}$ content ranges from $300 \mathrm{mg}$ to about $2 \mathrm{~g}$ in the human body[5]. High levels of vitamin $\mathrm{C}$ concentration are maintained in cells and tissues of a human. They are highest in the brain, eyes, leukocytes, adrenal, and pituitary gland. Relatively low levels of vitamin C concentrations are found in extracellular fluids, such as plasma, red blood cells, and saliva [5]. 
Fruits and vegetables are important sources of vitamin C, and $90 \%$ of the daily intake by humans comes from these sources. The concentration varies between different plant species. The animals are poor vitamin $C$ sources, and the level is usually $<30-40 \mathrm{mg} / 100 \mathrm{~g}$. Therefore plant sources become vital because of the high vitamin $C$ content, up to $5,000 \mathrm{mg} / 100 \mathrm{~g}$. In areas where vegetation is scanty, such as the cold arctic regions, people have traditionally depended on different sources of vitamin $C$, such as medicinal herbs (extracts from pine needles, rose hips, and tree barks) and animal organs, such as the raw liver and whale skin [31, 32]. Therefore, sufficient protein consumption from animal and vitamin $C$ from plant products is crucial for optimal development, growth, and health of humans [33]. The main objective of the study was to determine the vitamin $\mathrm{C}$ content in common edible food sources. The amount of vitamin $\mathrm{C}$ can be estimated by a redox titration with a standardized solution of iodine.

\section{Materials and Methods}

\subsection{Raw materials.}

The raw materials like lemon (Citrus limon), lime (Citrus aurantiifolia), navel orange (Citrus sinensis), grapevine (Vitis vinifera), fox grape (Vitis labrusca), pomegranate (Punica granatum), apple (Malus domestica), kiwifruit (Actinidia deliciosa), Indian tamarind (Tamarindus indica), holy basil (Ocimum tenuiflorum), coriander (Coriandrum sativum), tomato (Solanum lycopersicum), carrot (Daucus carota), radish (Raphanus sativus), bitter gourd (Momordica charantia), garlic (Allium sativum), onion (Allium cepa), potato (Solanum tuberosum), aubergine (Solanum melongena), bell pepper (Capsicum annuum), pea (Pisum sativum), common bean (Phaseolus vulgaris), pumpkin (Cucurbita moschata), pawpaw (Carica papaya), cucumber (Cucumis sativus), cabbage (Brassica oleracea var. capitata), and cauliflower (Brassica oleracea var. botrytis) were collected from a local market.

\subsection{Chemicals and reagents.}

The imperative chemicals and standard reagents used in the experiments were purchased from Sigma-Aldrich unless otherwise mentioned.

\subsection{Redox titration using iodine solution.}

This method determines the vitamin $\mathrm{C}$ concentration in a solution by a redox titration using iodine. Vitamin C, also known as ascorbic acid or L (+)-Ascorbic acid, belongs to the class of organic compounds called butenolides. Iodine is added in the course of titration, the ascorbic acid is oxidized to dehydroascorbic acid, while the iodine is reduced to iodide ions.

Ascorbic acid + Iodine $\left(\mathrm{I}_{2}\right) \rightarrow 2 \mathrm{I}^{-}+$Dehydroascorbic acid

Due to this, the iodine formed is instantly reduced to iodide as long as there is any ascorbic acid present in the solution. When all the ascorbic acid has been oxidized, the excess iodine is free to react with the starch indicator, forming a blue-black starch-iodine complex. This is the endpoint of the redox titration. This method is easier than the alternative method using potassium iodate solution. It is used for raw solid fruits and vegetables.

\subsection{Preparation of chemicals.}

\subsubsection{Preparation of iodine solution $\left(0.005 \mathrm{~mol} \mathrm{~L}^{-1}\right)$.}


$2 \mathrm{~g}$ of potassium iodide was taken in a $100 \mathrm{~mL}$ beaker, and $1.3 \mathrm{~g}$ of iodine crystal was added. Then $50 \mathrm{~mL}$ of distilled water was added and swirled for 20 minutes till the iodine was fully dissolved. The iodine solution was then transferred to a $1 \mathrm{~L}$ volumetric flask; after that, distilled water was added and made the solution up to $1 \mathrm{~L}$.

\subsubsection{Preparation of Starch indicator solution (5\%).}

$5 \mathrm{~g}$ of soluble starch was added to $100 \mathrm{~mL}$ of boiling distilled water in a conical flask by stirring. The solution was cooled down for use.

\subsection{Preparation of sample solution.}

$2 \mathrm{~mL}$ of the raw solution was pipetted in a conical flask, and $98 \mathrm{~mL}$ of distilled water was added to attain the diluted sample solution, i.e., 50 times diluted.

2.6. Estimation of vitamin C content by redox titration using iodine solution for common edible food sources.

$20 \mathrm{~mL}$ of the sample solution was pipetted into a $250 \mathrm{~mL}$ conical flask, and $150 \mathrm{~mL}$ of distilled water was added to it, followed by a $3 \mathrm{~mL}$ starch indicator solution. Then the sample solution was titrated with $0.005 \mathrm{~mol} \mathrm{~L}^{-1}$ iodine solution. The titration's endpoint was identified as the first permanent trace of a dark blue-black color due to the starch-iodide complex. The titration was repeated with further aliquots of sample solution until we obtained concordant results (Table 1).

\section{Results and Discussion}

\subsection{Calculation.}

Ascorbic acid $+\mathrm{I}_{2} \rightarrow 2 \mathrm{I}^{-}+$Dehydroascorbic Acid

As iodine solution is taken as the standard solution, it is taken as $0.005 \mathrm{~mol} \mathrm{~L}^{-1}$.

Molar ratio of iodine $=2 / 1=2$

First, calculate the moles of iodine using rule,

$\mathrm{N}=\mathrm{CV}$

Where, $\mathrm{C}=0.005 \mathrm{~mol} \mathrm{~L}^{-1}$

$\mathrm{V}=$ Mean value of the concordant readings

Then, calculate the moles of ascorbic acid (AA) in the given sample,

$\mathrm{N}_{\mathrm{AA}}=2 \mathrm{~N}$

Finally, convert the moles of AA found to mass

$\mathrm{M}=\mathrm{N}_{\mathrm{AA}} \times \mathrm{M}_{\mathrm{AA}}$

Where, $\mathrm{M}_{\mathrm{AA}}$ is the molecular mass of Ascorbic acid

Table 1. Mean value of different edible fruits and vegetables by redox titration method.

\begin{tabular}{l|l|l|l|l|l}
$\begin{array}{l}\text { Serial } \\
\text { number }\end{array}$ & Sample & $\begin{array}{l}\text { Initial burette } \\
\text { reading }\end{array}$ & $\begin{array}{l}\text { Final burette } \\
\text { reading }\end{array}$ & Difference & Mean \\
\hline 1 & Lemon (Citrus limon) & 13.0 & 14.8 & 1.8 & \\
& & 14.8 & 16.4 & 1.6 & \multirow{2}{*}{1.6} \\
& & 16.4 & 18.0 & 1.6 & \\
\hline 2 & Lime (Citrus aurantiifolia) & 18.0 & 19.6 & 1.6 & \\
& & 18.4 & 18.4 & 2.2 & 2.0
\end{tabular}




\begin{tabular}{|c|c|c|c|c|c|}
\hline $\begin{array}{l}\text { Serial } \\
\text { number }\end{array}$ & Sample & $\begin{array}{l}\text { Initial burette } \\
\text { reading }\end{array}$ & $\begin{array}{l}\text { Final burette } \\
\text { reading }\end{array}$ & Difference & Mean \\
\hline 3 & Navel orange (Citrus sinensis) & $\begin{array}{l}0.2 \\
2.2 \\
4.4 \\
6.6 \\
\end{array}$ & $\begin{array}{l}2.2 \\
4.4 \\
6.6 \\
8.8 \\
\end{array}$ & $\begin{array}{l}2.0 \\
2.2 \\
2.2 \\
2.2\end{array}$ & 2.2 \\
\hline 4 & Grapevine (Vitis vinifera) & $\begin{array}{l}4.4 \\
6.6 \\
8.8 \\
11.2 \\
\end{array}$ & $\begin{array}{l}6.6 \\
8.8 \\
11.2 \\
13.4 \\
\end{array}$ & $\begin{array}{l}2.2 \\
2.2 \\
2.4 \\
2.2 \\
\end{array}$ & 2.2 \\
\hline 5 & Fox grape (Vitis labrusca) & $\begin{array}{l}25.6 \\
27.8 \\
29.8 \\
32.0 \\
\end{array}$ & $\begin{array}{l}27.8 \\
29.8 \\
32.0 \\
34.2 \\
\end{array}$ & $\begin{array}{l}2.2 \\
2.0 \\
2.2 \\
2.2 \\
\end{array}$ & 2.2 \\
\hline 6 & $\begin{array}{l}\text { Pomegranate } \\
\text { granatum) }\end{array}$ & $\begin{array}{l}30.0 \\
31.2 \\
32.2 \\
33.2 \\
\end{array}$ & $\begin{array}{l}31.2 \\
32.2 \\
33.2 \\
34.2 \\
\end{array}$ & $\begin{array}{l}1.2 \\
1.0 \\
1.0 \\
1.0 \\
\end{array}$ & 1.0 \\
\hline 7 & Apple (Malus domestica) & $\begin{array}{l}34.2 \\
36.2 \\
38.0 \\
40.0\end{array}$ & $\begin{array}{l}36.2 \\
38.0 \\
40.0 \\
42.0\end{array}$ & $\begin{array}{l}2.0 \\
1.8 \\
2.0 \\
2.0\end{array}$ & 2.0 \\
\hline 8 & Kiwifruit (Actinidia deliciosa) & $\begin{array}{l}35.8 \\
39.0 \\
41.4 \\
43.8 \\
\end{array}$ & $\begin{array}{l}39.0 \\
41.4 \\
43.8 \\
46.2 \\
\end{array}$ & $\begin{array}{l}2.2 \\
2.4 \\
2.4 \\
2.4 \\
\end{array}$ & 2.4 \\
\hline 9 & Pawpaw (Carica papaya) & $\begin{array}{l}17.2 \\
19.4 \\
21.8 \\
24.0 \\
\end{array}$ & $\begin{array}{l}19.4 \\
21.8 \\
24.0 \\
26.2 \\
\end{array}$ & $\begin{array}{l}2.2 \\
2.4 \\
2.2 \\
2.2 \\
\end{array}$ & 2.2 \\
\hline 10 & $\begin{array}{l}\text { Indian tamarind (Tamarindus } \\
\text { indica) }\end{array}$ & $\begin{array}{l}40.2 \\
46.8 \\
50.2 \\
52.0\end{array}$ & $\begin{array}{l}42.0 \\
49.4 \\
52.0 \\
53.8\end{array}$ & $\begin{array}{l}1.8 \\
1.6 \\
1.8 \\
1.8\end{array}$ & 1.8 \\
\hline 11 & $\begin{array}{l}\text { Holy basil } \quad \text { (Ocimum } \\
\text { tenuiflorum })\end{array}$ & $\begin{array}{l}58.0 \\
60.4 \\
63.0 \\
65.4 \\
\end{array}$ & $\begin{array}{l}60.4 \\
63.0 \\
65.4 \\
67.8 \\
\end{array}$ & $\begin{array}{l}2.4 \\
2.6 \\
2.4 \\
2.4 \\
\end{array}$ & 2.4 \\
\hline 12 & $\begin{array}{l}\text { Coriander } \\
\text { sativum })\end{array}$ & $\begin{array}{l}20.8 \\
23.2 \\
25.2 \\
27.2 \\
\end{array}$ & $\begin{array}{l}23.2 \\
25.2 \\
27.2 \\
29.2 \\
\end{array}$ & $\begin{array}{l}2.4 \\
2.0 \\
2.0 \\
2.0 \\
\end{array}$ & 2.0 \\
\hline 13 & Carrot (Daucus carota) & $\begin{array}{l}22.2 \\
23.8 \\
25.6 \\
27.2\end{array}$ & $\begin{array}{l}23.8 \\
25.6 \\
27.2 \\
28.8\end{array}$ & $\begin{array}{l}1.6 \\
1.8 \\
1.6 \\
1.6\end{array}$ & 1.6 \\
\hline 14 & Radish (Raphanus sativus) & $\begin{array}{l}23.6 \\
25.6 \\
27.8 \\
30.2 \\
\end{array}$ & $\begin{array}{l}25.6 \\
27.8 \\
30.0 \\
32.4 \\
\end{array}$ & $\begin{array}{l}2.0 \\
2.2 \\
2.2 \\
2.2 \\
\end{array}$ & 2.2 \\
\hline 15 & Garlic (Allium sativum) & $\begin{array}{l}25.4 \\
28.0 \\
30.2 \\
32.4 \\
\end{array}$ & $\begin{array}{l}28.0 \\
30.2 \\
32.4 \\
36.6\end{array}$ & $\begin{array}{l}2.6 \\
2.2 \\
2.2 \\
2.2\end{array}$ & 2.2 \\
\hline 16 & Onion (Allium cepa) & $\begin{array}{l}25.0 \\
26.8 \\
28.4 \\
30.0 \\
\end{array}$ & $\begin{array}{l}26.8 \\
28.4 \\
30.0 \\
31.6 \\
\end{array}$ & $\begin{array}{l}1.8 \\
1.6 \\
1.6 \\
1.6\end{array}$ & 1.6 \\
\hline 17 & Potato (Solanum tuberosum) & $\begin{array}{l}14.4 \\
16.4 \\
18.8 \\
20.6 \\
\end{array}$ & $\begin{array}{l}16.4 \\
18.8 \\
20.6 \\
22.4 \\
\end{array}$ & $\begin{array}{l}2.0 \\
1.8 \\
1.8 \\
1.8 \\
\end{array}$ & 1.8 \\
\hline 18 & $\begin{array}{l}\text { Tomato } \\
\text { lycopersicum) }\end{array}$ & $\begin{array}{l}20.4 \\
22.0 \\
23.4 \\
25.0\end{array}$ & $\begin{array}{l}22.0 \\
23.4 \\
25.0 \\
26.6\end{array}$ & $\begin{array}{l}1.6 \\
1.4 \\
1.6 \\
1.6\end{array}$ & 1.6 \\
\hline 19 & $\begin{array}{ll}\text { Aubergine } & \text { (Solanum } \\
\text { melongena }) & \end{array}$ & $\begin{array}{l}28.4 \\
30.6 \\
32.6 \\
34.6\end{array}$ & $\begin{array}{l}30.6 \\
32.6 \\
34.6 \\
36.6\end{array}$ & $\begin{array}{l}2.2 \\
2.0 \\
2.0 \\
2.0\end{array}$ & 2.0 \\
\hline
\end{tabular}


https://doi.org/10.33263/LIANBS103.23612369

\begin{tabular}{|c|c|c|c|c|c|}
\hline $\begin{array}{l}\text { Serial } \\
\text { number }\end{array}$ & Sample & $\begin{array}{l}\text { Initial burette } \\
\text { reading }\end{array}$ & $\begin{array}{l}\text { Final burette } \\
\text { reading }\end{array}$ & Difference & Mean \\
\hline 20 & $\begin{array}{l}\text { Bell pepper (Capsicum } \\
\text { annuиm) }\end{array}$ & $\begin{array}{l}7.2 \\
9.6 \\
11.8 \\
14.0\end{array}$ & $\begin{array}{l}9.6 \\
11.8 \\
14.0 \\
16.2\end{array}$ & $\begin{array}{l}2.4 \\
2.2 \\
2.2 \\
2.2\end{array}$ & 2.2 \\
\hline 21 & Pea (Pisum sativum) & $\begin{array}{l}32.8 \\
35.2 \\
37.6 \\
39.8 \\
\end{array}$ & $\begin{array}{l}35.2 \\
37.6 \\
39.8 \\
42.2 \\
\end{array}$ & $\begin{array}{l}2.4 \\
2.4 \\
2.2 \\
2.4\end{array}$ & 2.4 \\
\hline 22 & $\begin{array}{l}\text { Common bean (Phaseolus } \\
\text { vulgaris) }\end{array}$ & $\begin{array}{l}14.6 \\
16.2 \\
17.6 \\
19.0\end{array}$ & $\begin{array}{l}16.2 \\
17.6 \\
19.0 \\
20.4\end{array}$ & $\begin{array}{l}1.6 \\
1.4 \\
1.4 \\
1.4\end{array}$ & 1.4 \\
\hline 23 & $\begin{array}{l}\text { Bitter gourd (Momordica } \\
\text { charantia) }\end{array}$ & $\begin{array}{l}13.2 \\
15.6 \\
18.2 \\
20.8\end{array}$ & $\begin{array}{l}15.6 \\
18.2 \\
20.8 \\
23.4\end{array}$ & $\begin{array}{l}2.4 \\
2.6 \\
2.6 \\
2.6\end{array}$ & 2.6 \\
\hline 24 & $\begin{array}{ll}\text { Pumpkin } & \text { (Cucurbita } \\
\text { moschata) }\end{array}$ & $\begin{array}{l}21.6 \\
24.2 \\
26.2 \\
28.4 \\
\end{array}$ & $\begin{array}{l}23.8 \\
26.2 \\
28.4 \\
30.6 \\
\end{array}$ & $\begin{array}{l}2.2 \\
2.0 \\
2.2 \\
2.2 \\
\end{array}$ & 2.2 \\
\hline 25 & Cucumber (Cucumis sativus) & $\begin{array}{l}9.0 \\
10.0 \\
11.2 \\
12.4\end{array}$ & $\begin{array}{l}10.0 \\
11.2 \\
12.4 \\
13.6\end{array}$ & $\begin{array}{l}1.0 \\
1.2 \\
1.2 \\
1.2\end{array}$ & 1.2 \\
\hline 26 & $\begin{array}{l}\text { Cabbage (Brassica oleracea } \\
\text { var. capitata) }\end{array}$ & $\begin{array}{l}21.2 \\
23.2 \\
25.0 \\
26.8\end{array}$ & $\begin{array}{l}23.2 \\
25.0 \\
26.8 \\
28.6\end{array}$ & $\begin{array}{l}2.0 \\
1.8 \\
1.8 \\
1.8\end{array}$ & 1.8 \\
\hline 27 & $\begin{array}{l}\text { Cauliflower (Brassica } \\
\text { oleracea var. botrytis) }\end{array}$ & $\begin{array}{l}8.8 \\
11.4 \\
13.8 \\
16.2\end{array}$ & $\begin{array}{l}11.4 \\
13.8 \\
16.2 \\
18.6\end{array}$ & $\begin{array}{l}2.6 \\
2.4 \\
2.4 \\
2.4\end{array}$ & 2.4 \\
\hline
\end{tabular}

Table 2. Analysis of different concentrations of vitamin $\mathrm{C}$ in edible food samples by redox titration using iodine solution.

\begin{tabular}{|c|c|c|}
\hline $\begin{array}{l}\text { Serial } \\
\text { number }\end{array}$ & Sample & Amount of vitamin $C(\mathbf{m g} / \mathbf{1 0 0 g})$ \\
\hline 1 & Lemon (Citrus limon) & 18.73 \\
\hline 2 & Lime (Citrus aurantiifolia) & 15.05 \\
\hline 3 & Navel orange (Citrus sinensis) & 30.69 \\
\hline 4 & Grapevine (Vitis vinifera) & 24.81 \\
\hline 5 & Fox grape (Vitis labrusca) & 21.03 \\
\hline 6 & Pomegranate (Punica granatum) & 11.54 \\
\hline 7 & Apple (Malus domestica) & 4.00 \\
\hline 8 & Kiwifruit (Actinidia deliciosa) & 22.25 \\
\hline 9 & Pawpaw (Carica papaya) & 45.37 \\
\hline 10 & Indian tamarind (Tamarindus indica) & 89.55 \\
\hline 11 & Holy basil (Ocimum tenuiflorum) & 39.61 \\
\hline 12 & Coriander (Coriandrum sativum) & 63.20 \\
\hline 13 & Carrot (Daucus carota) & 33.14 \\
\hline 14 & Radish (Raphanus sativus) & 35.70 \\
\hline 15 & Garlic (Allium sativum) & 40.95 \\
\hline 16 & Onion (Allium cepa) & 30.79 \\
\hline 17 & Potato (Solanum tuberosum) & 33.65 \\
\hline 18 & Tomato (Solanum lycopersicum) & 16.47 \\
\hline 19 & Aubergine (Solanum melongena) & 35.96 \\
\hline 20 & Bell pepper (Capsicum annuum) & 45.58 \\
\hline 21 & Pea (Pisum sativum) & 50.84 \\
\hline 22 & Common bean (Phaseolus vulgaris) & 41.28 \\
\hline 23 & Bitter gourd (Momordica charantia) & 67.60 \\
\hline 24 & Pumpkin (Cucurbita moschata) & 36.30 \\
\hline 25 & Cucumber (Cucumis sativus) & 24.23 \\
\hline 26 & Cabbage (Brassica oleracea var. capitata) & 43.25 \\
\hline 27 & Cauliflower (Brassica oleracea var. botrytis) & 28.86 \\
\hline
\end{tabular}




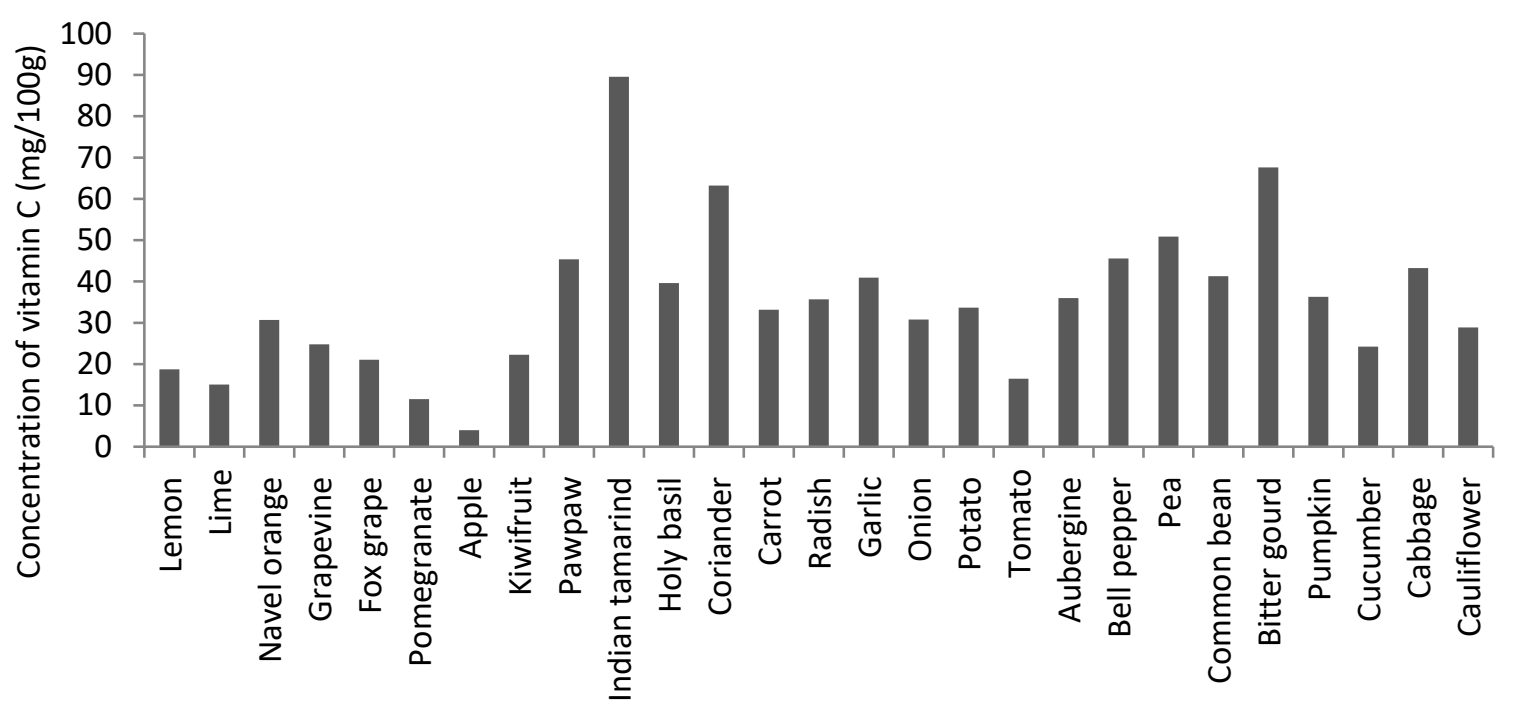

Figure 1. Different concentrations of vitamin C present in common edible food sources.

The vitamin $\mathrm{C}$ concentration per 100 gram lemon (Citrus limon) is $18.73 \mathrm{mg}$, lime (Citrus aurantiifolia) is $15.05 \mathrm{mg}$, navel orange (Citrus sinensis) is $30.69 \mathrm{mg}$, grapevine (Vitis vinifera) is $24.81 \mathrm{mg}$, fox grape (Vitis labrusca) is $21.03 \mathrm{mg}$, pomegranate (Punica granatum) is $11.54 \mathrm{mg}$, apple (Malus domestica) is $4.0 \mathrm{mg}$, kiwifruit (Actinidia deliciosa) is $22.25 \mathrm{mg}$, pawpaw (Carica papaya) is $45.37 \mathrm{mg}$, Indian tamarind (Tamarindus indica) is $89.55 \mathrm{mg}$, holy basil (Ocimum tenuiflorum) is $39.61 \mathrm{mg}$, coriander (Coriandrum sativum) is $63.20 \mathrm{mg}$, carrot (Daucus carota) is $33.14 \mathrm{mg}$, radish (Raphanus sativus) is $35.70 \mathrm{mg}$, garlic (Allium sativum) is $40.95 \mathrm{mg}$, onion (Allium cepa) is $30.79 \mathrm{mg}$, potato (Solanum tuberosum) is $33.65 \mathrm{mg}$, tomato (Solanum lycopersicum) is $16.47 \mathrm{mg}$, aubergine (Solanum melongena) is $35.96 \mathrm{mg}$, bell pepper (Capsicum annuum) is $45.58 \mathrm{mg}$, pea (Pisum sativum) is $50.84 \mathrm{mg}$, common bean (Phaseolus vulgaris) is $41.28 \mathrm{mg}$, bitter gourd (Momordica charantia) is $67.60 \mathrm{mg}$, pumpkin (Cucurbita moschata) is $36.30 \mathrm{mg}$, cucumber (Cucumis sativus) is $24.23 \mathrm{mg}$, cabbage (Brassica oleracea var. capitata) is $43.25 \mathrm{mg}$, and cauliflower (Brassica oleracea var. botrytis) is $28.86 \mathrm{mg}$ respectively (Table 2). It is evident from the present findings that the concentration of vitamin $\mathrm{C}$ varies from $4.00 \mathrm{mg} / 100 \mathrm{~g}$ to $89.55 \mathrm{mg} / 100 \mathrm{~g}$ (Figure 1). Vitamin C concentration is found to be highest in Indian tamarind (Tamarindus indica) is $89.55 \mathrm{mg}$.

\section{Conclusions}

From this experiment, it was concluded that Indian tamarind had more vitamin $\mathrm{C}$ content than lemon, lime, navel orange, grapevine, fox grape, pomegranate, apple, kiwifruit, pawpaw, holy basil, coriander, carrot, radish, garlic, onion, potato, tomato, aubergine, bell pepper, pea, common bean, bitter gourd, pumpkin, cucumber, cabbage, and cauliflower. A further detailed and comprehensive evaluation is suggested to be undertaken on these samples considering vitamin $\mathrm{C}$ concentration because these food sources are easily available in the local market, which would help improve immunity for diseases.

\section{Funding}

This research received no external funding.

\section{Acknowledgments}


This research has no acknowledgment.

\section{Conflicts of Interest}

The authors declare no conflict of interest.

\section{References}

1. Haworth, W.N. The constitution of ascorbic acid. J. Chem. Technol. Biotechnol. 1933, 52, 482-485.

2. Li, Y.; Schellhorn, H.E. New developments and novel therapeutic perspectives for vitamin C. J Nutr. 2007, 137, 10, 2171-2184.

3. Carr, A.C.; Frei, B. Toward a new recommended dietary allowance for vitamin C based on antioxidant and health effects in humans. Am J Clin Nutr 1999, 69, 1086-107, https://doi.org/10.1093/ajcn/69.6.1086.

4. Frei, B.; England, L.; Ames, B.N. Ascorbate is an outstanding antioxidant in human blood plasma. Proc Natl Acad Sci. 1989, 86, 6377-81, https://doi.org/10.1073/pnas.86.16.6377.

5. Jacob, R.A.; Sotoudeh, G. Vitamin C function and status in chronic disease. Nutr Clin Care 2002, 5, 66-74, https://doi.org/10.1046/j.1523-5408.2002.00005.x.

6. Block, G.; Patterson, B.; Subar, A. Fruit, vegetables, and cancer prevention: a review of the epidemiological evidence. Nutr Cancer 1992, 18, 1-29, https://doi.org/10.1080/01635589209514201.

7. Steinmetz, K.A.; Potter, J.D. Vegetables, fruit, and cancer prevention: a review. J Am Diet Assoc. 1996, 96, 1027-1039, https://doi.org/10.1016/S0002-8223(96)00273-8.

8. Gershoff, S.N. Vitamin C (ascorbic acid): new roles, new requirements? Nutr Rev 1993, 51, 313-26, https://doi.org/10.1111/j.1753-4887.1993.tb03757.x.

9. Weinstein, M.; Babyn, P.; Zlotkin, S. An orange a day keeps the doctor away: scurvy in the year 2000. Pediatrics 2001,108, https://doi.org/10.1542/peds.108.3.e55.

10. Wang, A.H.; Still, C. Old world meets modern: a case report of scurvy. Nutr Clin Pract 2007, 22, 445-8, https://doi.org/10.1177/0115426507022004445.

11. Institute of Medicine (US) Panel on Dietary Antioxidants and Related Compounds. In: Dietary Reference Intakes for Vitamin C, Vitamin E, Selenium, and Carotenoids. National Academies Press (US), 2000, https://doi.org/10.17226/9810.

12. Stephen, R.; Utecht, T. Scurvy identified in the emergency department: a case report. J Emerg Med 2001, 21, 235-7, https://doi.org/10.1016/s0736-4679(01)00377-8.

13. Pauling, L. Ascorbic Acid and the Common Cold. Scottish Medical Journal 1973, 18, 1-2.

14. Galmés, S.; Serra, F.; Palou, A. Current State of Evidence: Influence of Nutritional and Nutrigenetic Factors on Immunity in the COVID-19 Pandemic Framework. Nutrient 2020,12, https://doi.org/10.3390/nu12092738.

15. Feyaerts, A.F.; Luyten, W. Vitamin C as prophylaxis and adjunctive medical treatment for COVID-19? Nutrition 2020, 79, https://doi.org/10.1016/j.nut.2020.110948.

16. Arabi, Y M.; Fowler, R.; Hayden, F.G. Critical care management of adults with community-acquired severe respiratory viral infection. Intensive care medicine 2020, 46, 315-328, https://doi.org/10.1007/s00134-02005943-5.

17. Hemilä, H. Vitamin C and SARS coronavirus. Journal of Antimicrobial Chemotherapy 2003, 52, 1049-1050, https://doi.org/10.1093/jac/dkh002.

18. Hemilä, H. Vitamin $\mathrm{C}$ intake and susceptibility to pneumonia. The Pediatric infectious disease journal 1997, 16, 836-837.

19. Hemilä, H. Vitamin C intake and susceptibility to the common cold. British Journal of Nutrition 1997, 77 , 59-72, https://doi.org/10.1017/S0007114500002889.

20. Atherton, J.G.; Kratzing, C.C.; Fisher, A. The effect of ascorbic acid on infection of chick-embryo ciliated tracheal organ cultures by coronavirus.Archives of virology 1978,56, 195199,https://doi.org/10.1007/BF01317848.

21. Leibovitz, B.; Siegel, B.V. Ascorbic acid and the immune response. Adv Exp Med Biol. 1981, 135, 125,https://doi.org/10.1007/978-1-4615-9200-6_1.

22. Hemilä, H. Vitamin C, respiratory infections and the immune system. Trends Immunol. 2003, 24, 579-580, https://doi.org/10.1016/j.it.2003.09.004.

23. Chambial, S.; Dwivedi, S.; Shukla, K.K.; John, P.J.; Sharma, P. Vitamin C in disease prevention and cure: an overview. Indian J Clin Biochem. 2013, 28, 314-328,https://doi.org/10.1007/s12291-013-0375-3.

24. Özkaya, D.; Naziroğlu, M.; Armağan, A.; Demirel, A.; Köroglu, B.K.; Çolakoğlu, N.; Kükner, A.; Sönmez, T.T. Dietary vitamin C and E modulates oxidative stress induced-kidney and lens injury in diabetic aged male rats through modulating glucose homeostasis and antioxidant systems. Cell Biochem Funct. 2011, 29, 287-293,https://doi.org/10.1002/cbf.1749. 
25. Naziroğlu, M.; Butterworth, P.J.; Sonmez, T.T. Dietary vitamin C and E modulates antioxidant levels in blood, brain, liver, muscle, and testes in diabetic aged rats. Int J Vit Nutr Res. 2011, 81, 347-357, https://doi.org/10.1024/0300-9831/a000083.

26. Shaban El-Neweshy, M.; Said El-Sayed, Y. Influence of vitamin C supplementation on lead-induced histopathological alterations in male rats. Exp Toxicol Pathol. 2011, 63, 221-227, https://doi.org/10.1016/j.etp.2009.12.003.

27. Dakhale, G.N.; Khanzode, S.D.; Khanzode, S.S.; Saoji, A. Supplementation of vitamin C with atypical antipsychotics reduces oxidative stress and improves the outcome of schizophrenia. Psychopharmacology 2005, 182,494-498, https://doi.org/10.1007/s00213-005-0117-1.

28. Patumraj, S.; Wongeakin, N.; Sridulyakul, P.; Jariyapongskul, A.; Futrakul, N.; Bunnag, S. Combined effects of curcumin and vitamin $\mathrm{C}$ to protect endothelial dysfunction in the iris tissue of STZ-induced diabetic rats.Clinical hemorheology and microcirculation 2006, 35, 481-489.

29. Satpathy, L.; Parida, S.P. Acute Toxicity Assessment and Behavioral Responses Induced by Kandhamal haladi in Adult Zebrafish (Danio rerio). Biointerface Research in Applied Chemistry 2020, 11, 7368-7381, https://doi.org/10.33263/BRIAC111.73687381.

30. Padayatty, S.J.; Sun, H.; Wang, Y.; Riordan, H.D.; Hewitt, S.M.; Katz, A.; Wesley, R.A.; Levine, M. Vitamin C pharmacokinetics: implications for oral and intravenous use. Ann Intern Med 2004, 140, 533-537, https://doi.org/10.7326/0003-4819-140-7-200404060-00010.

31. Stevenson, N.R.; Brush, M.K. Existence and characteristic of $\mathrm{Na}^{+}$dependent active transport of ascorbic acid in guinea pigs. Am J Clin Nutr. 1969, 22, https://doi.org/10.1093/ajcn/22.3.318.

32. Malo, C.; Wilson, J.X. Glucose modulates vitamin $\mathrm{C}$ transport in adult human small intestinal brush border membrane vesicles. J Nutr. 2000, 130, 63-69, https://doi.org/10.1093/jn/130.1.63.

33. Satpathy, L.; Dash, D.; Sahoo, P.; Anwar, T.S.; Parida, S.P. Quantitation of Total Protein Content in Some Common Edible Food Sources by Lowry Protein Assay. Letters in Applied NanoBioScience 2020, 9, 12751283, https://doi.org/10.33263/LIANBS93.12751283. 